\title{
Jean-Louis Cabanès - Le Journal des Goncourt
}

Entretien avec Françoise Simonet-Tenant

\section{Françoise Simonet-Tenant}

\section{(2) OpenEdition}

Journals

Édition électronique

URL : http://journals.openedition.org/genesis/507

DOI : $10.4000 /$ genesis. 507

ISSN : 2268-1590

Éditeur :

Presses universitaires de Paris Sorbonne (PUPS), Société internationale de génétique artistique littéraire et scientifique (SIGALES)

\section{Édition imprimée}

Date de publication : 1 janvier 2011

Pagination : 127-131

ISBN : 978-2-84050-749-9

ISSN : 1167-5101

\section{Référence électronique}

Françoise Simonet-Tenant, « Jean-Louis Cabanès - Le Journal des Goncourt », Genesis [En ligne], 32 | 2011, mis en ligne le 17 mai 2013, consulté le 19 avril 2019. URL : http://journals.openedition.org/ genesis/507; DOI : 10.4000/genesis.507 


\title{
Le Journal des Goncourt Entretien avec Françoise Simonet-Tenant
}

\author{
Jean-Louis Cabanès
}

Françoise Simonet-Tenant - En 2005 a été publié sous votre direction le tome I d' une nouvelle édition du Journal des Goncourt ${ }^{1}$. Quelles raisons vous ont amené à préparer cette nouvelle édition après celle publiée du vivant d'Edmond (premier volume publié en $1887 \mathrm{chez}$ Charpentier) et celle réalisée par Robert Ricatte (Fasquelle et Flammarion, 1956 ; Robert Laffont, 1989)?

Jean-Louis Cabanès - Au départ nous étions partis sur l'idée d'une édition électronique. L'éditeur ayant fait défaut, nous sommes revenus à l'idée d'une édition critique sur papier sans mesurer la tâche qui nous attendait. Le travail de bénédictin mené par Robert Ricatte pour établir le texte, aussi énorme qu'il fût, a été réalisé au début des années cinquante, et les principes d'édition d'aujourd'hui ne sont plus exactement les mêmes. Nous nous sommes ainsi heurtés à des problèmes de découpages de paragraphes et de ponctuation. Nous nous sommes efforcés de restituer une ponctuation fidèle au manuscrit, même si c'est parfois difficile : il arrive qu'il n'y ait pas de ponctuation chez Edmond de Goncourt ; il arrive aussi que les points aient valeur de virgule ou que la ponctuation n'ait pas de valeur discriminatoire. Nous avons renoncé au tressage opéré occasionnellement par Robert Ricatte entre le manuscrit et l'édition publiée du vivant d'Edmond de Goncourt. Il fallait être respectueux des tirets, diminuer la part des points d'exclamation qui surabondent dans l'édition Ricatte, conserver souvent les points-virgules dans les descriptions ou les périodes éloquentes. Comme le manuscrit du journal d'une part et le texte publié du vivant des Goncourt d'autre part nous semblent constituer deux œuvres distinctes, nous avons publié en annexe de notre édition le texte de l'édition Charpentier, ce qui donne au lecteur la possibilité de comparer les deux textes, principe que nous allons poursuivre dans les tomes suivants. Nous avons également souhaité éclairer le texte par une annotation copieuse. Chaque tome de notre édition sera, en outre, accompagné d'un dictionnaire ou répertoire qui comprend à la fois les acteurs du champ artistique et littéraire, les figures mythiques du XIX ${ }^{\mathrm{e}}$ siècle, les revues et périodiques, les lieux de sociabilité mais aussi les lieux institutionnels de la misère (prisons, hôpitaux, maisons de prostitution). C'est une sorte de panorama de la vie culturelle, et sociale, une petite encyclopédie portative que l'on a voulu établir à partir du Journal des Goncourt.

F. S.-T. - Une telle édition est une lourde tâche. Comment le travail s'est-il réparti entre les différents membres de l'équipe?

J.-L. C. - J'ai assumé avec mon épouse le travail d'établissement du texte. L'annotation a nécessité la formation d'une équipe qui comprenait à la fois des spécialistes d'histoire de l'art, du réalisme et du naturalisme, de la presse, des lieux de sociabilité, de la correspondance, de la bohème littéraire. Il sera sans doute nécessaire de faire appel pour les tomes suivants à des japonisants. Les historiens nous font défaut.

F. S.-T. - Comment se présente matériellement le manuscrit? Y a-t-il beaucoup de ratures, trouve-t-on des collages?

J.-L. C. - Le Journal comprend onze cahiers (huit in-octavo et trois in-quarto) reliés en vert foncé qui ne sont pas tous de la même épaisseur. On observe jusqu'en avril 1855 une grande

1. Journal des Goncourt, t. I, 1851-1857, édition critique publiée sous la direction de Jean-Louis Cabanès, Paris, Champion, 2005. Depuis la date de cet entretien, réalisé en janvier 2008, a paru, sous la direction de JeanLouis Cabanès le Journal des Goncourt, tome II, 1858-1860 (Paris, Champion, 2008). 
présence de collages qui se poursuivent d'ailleurs en quantité moindre au-delà de cette date. Il serait sans doute nécessaire d'établir une typologie de ces collages. Le journal ne trouve sa véritable allure qu'au milieu de l'année 1855, comme le constatait d'ailleurs Edmond lui-même, et comme Robert Ricatte l'a rappelé. En ce qui concerne l'organisation de l'écrit sur la page, on note l'existence de marges dans lesquelles on trouve parfois des adjonctions qui peuvent être accompagnées de flèches. Parfois, un paragraphe est entouré d'un trait, accompagné d'une flèche injonctive imposant qu'il soit transféré à un autre moment du texte, daté parfois d'un autre jour. Les commentaires à teneur métadiscursive sont peu fréquents. Nous avons pris le parti de signaler exhaustivement les ratures, alors que l'édition Ricatte en avait fait basculer quelques-unes au sein même du texte et ne les avait pas toutes relevées. Ces ratures obéissent parfois à une volonté de censure, parfois à des motifs esthétiques. Je parlerais également dans certains cas de « ratures duplices » dans la mesure où certains passages biffés sont aisés à déchiffrer par l'éditeur alors que d'autres ont été rendus intentionnellement illisibles.

F. S.-T. - Qu'en est-il de la datation des entrées, pierre angulaire du journal?

J.-L. C. - La datation est plus complexe qu'on pourrait le penser. Par exemple une date est parfois raturée et remplacée par une autre. Parfois encore, le Journal procède, si l'on peut dire, par bonds et par retours. Ainsi nous passons du 25 décembre 1864 au 28 janvier 1865. Or tout le passage écrit à cette dernière date est raturé. Le journal se poursuit ensuite comme si de rien n'était : 1er janvier, 5 janvier, etc. jusqu'au... 28 janvier où reparaît intégralement le texte écrit à la date du 28 janvier et dont je viens de dire qu'il avait été précédemment raturé. La chronologie cesse, à l'occasion, d'être linéaire, ce qui n'est pas sans poser de problème à l'éditeur. Ainsi, toujours pour l'année 1864 , je relève, en suivant l'ordre du manuscrit, les dates suivantes : 26 septembre, 30 septembre, 27 septembre, 20 octobre, 3 octobre... Cette manipulation du temps suggère de façon évidente la volonté de faire œuvre à partir du journal. Mais elle n'est pas la règle. Encore une fois, il faut prendre acte de la complexité d'une écriture fidèle le plus souvent au déroulement chronologique, mais néanmoins occasionnellement brouillée.
F. S.-T. - Il y a, tout au moins jusqu'à la mort de Jules en 1870, deux scribes. Créer à deux, écrire à quatre mains : la création en collaboration suppose des processus génétiques et des enjeux esthétiques singuliers. Comment se répartit l'écriture quand les deux frères tiennent le journal? Est-il facile pour le lecteur du texte manuscrit de distinguer aisément les deux graphies? Peut-on véritablement prendre mesure de la complexité de l'écriture duelle? Le choix du pronom personnel est parfois déroutant, comme dans l'entrée du 6 janvier 1857 : peut-on établir des usages réguliers dans le choix de l'un ou l'autre des deux pronoms?

J.-L. C. - Les écritures des deux frères sont très différentes : celle de Jules est anguleuse, resserrée, petite, celle d'Edmond arrondie et toute en courbes. Les deux écritures se laissent déchiffrer. Paradoxalement c'est parfois la propreté de l'écriture qui pose problème, la page impeccablement calligraphiée suggère qu'il y a eu, peut-être, copie d'un texte antérieur : je pense en particulier à l'ouverture du journal, à l'entrée du 2 décembre 1851. Jusque vers 1854-1855 les deux frères se succèdent quasi alternativement comme scripteurs, sans que cette alternance signifie une répartition des tâches; en revanche, par la suite, c'est massivement Jules qui est le scribe. Jules semble celui qui écrit et qui parle (souvent) pour deux, même si, à en croire Judith Gautier, dans la conversation courante, Edmond utilise un «nous », alors que Jules s'énonce à la première personne. Il y a une entrée très troublante sur un rêve, daté du dimanche 18 novembre 1860 . Ce rêve, dans le manuscrit, est écrit à deux, et il n'y a pourtant, bien évidemment qu'un seul rêveur. Jules prend d'abord la plume, viennent ensuite des lignes écrites par Edmond, mais elles sont rapportées sur le cahier par le truchement d'un collage.

\section{F. S.-T. - Le Journal des Goncourt se fait-il polyphonique par l'insertion de lettres citées ou recopiées?}

J.-L. C. - Oui, beaucoup de lettres reçues sont recopiées. Généralement le recopiage n'est pas intégral. Il arrive également que le Journal puisse accueillir un brouillon : je pense en particulier à la lettre adressée par les deux frères au garde des Sceaux pour protester contre l'usurpation de leur nom et revendiquer l'usage exclusif du titre nobiliaire 
« de Goncourt ». Cette lettre datée du 22 avril 1860, copieusement raturée, est insérée dans le manuscrit, entre deux folios et au milieu d'une phrase.

F. S.-T. - Au-delà de la pratique de l'insertion des lettres, la question de la présence de la voix d'autrui dans le Journal semble importante. Dans quelle mesure l'écriture diariste devient-elle chez les frères Goncourt une écriture sténographique?

J.-L. C. - Oui, le Journal entend restituer la parole d'autrui (les diaristes rapportent notamment les conversations de Sainte-Beuve, de Gautier, de Renan, de Taine, de Flaubert) et il y a un souci très net d'oralité. Edmond prétend que la retranscription des paroles d'autrui se faisait dans un laps de temps très court. On ne sait s'il possédait des carnets de notes. J'en doute. L'oralité est un critère. Diderot, Michelet sont appréciés parce que leur style tient d'une langue écrite parlée.

F. S.-T. - N'est-il pas paradoxal que des écrivains tels que les Goncourt qui se réclamaient de l'écriture-artiste - or il n'est rien de moins spontané que l'écriture-artiste - se soient adonnés à une écriture diariste qui accorde une large place à l'oralité ?

J.-L. C. - La contradiction n'est qu'apparente. Il ne faut pas oublier que la question de la voix reste posée, dans l'écriture-artiste, par les procédés d'emphase et de reprise. L'écriture-artiste ne se réduit pas à un sertissage d'expressions précieuses, elle répond à un souci d'éloquence et, sous des formes diverses, d'insistance, d'accentuation, de subjectivation d'un discours.

\section{F. S.-T. - Les diaristes Goncourt ont-ils des modèles d'écriture?}

J.-L. C. - Ce n'est qu'à partir de la deuxième moitié du $\mathrm{XIX}^{\mathrm{e}}$ siècle que commencent les premières publications de journaux intimes. Les Goncourt ont lu le Journal d'un poète de Vigny ; Edmond fera allusion au journal de Marie Bashkirtseff. Ils sont avant tout des lecteurs de mémoires : Saint-Simon, Chateaubriand sont des auteurs qu'ils aiment.
F. S.-T. - Y a-t-il plusieurs époques dans le Journal ? Pourquoi avoir arrêté ce tome I en 1857 ? La datation est très relâchée jusqu'en 1855, et on a l'impression que le journal prend vraiment et seulement sa vitesse de croisière à partir de cette date. Impression ou illusion?

J.-L.C. - Quand commence l'expérience diariste ? On peut rappeler l'existence de notes prises par Jules le 15 mai 1848. Elles sont intitulées par Edmond « Notes anciennes retrouvées ». Il faut tenir compte également du carnet de voyage écrit pendant un voyage en Algérie en 1849. Les Goncourt décident de donner à leur journal comme entrée inaugurale celle du 2 décembre 1851, mais le voyage en Algérie constitue un apprentissage de l'écriture diariste. Sur le plan du manuscrit l'on observe que l'entrée du 2 décembre 1851 est directement écrite dans un cahier qui, par ailleurs, comporte essentiellement des papiers collés parmi lesquels bon nombre de notes sur Gavarni. Dans les premières années du journal, peu de notes sont couchées directement sur les cahiers, ce qui donne donc un statut particulier à cette entrée du 2 décembre 1851. Il s'agit surtout au début d'un journal-album, où le collage tient une place essentielle. À mon sens, chez les Goncourt, l'activité de collectionneur n'est pas dissociable de celle de diariste : on collectionne des paroles, des portraits d'êtres... Le choix du 2 décembre 1851 pour l'entrée inaugurale n'est pas un hasard : c'est également celle du coup d'État sur lequel le texte pose un regard distant et ironique. Il y a de l'ironie à commencer un journal intime quand le pays est victime d'un coup d'État. L'écriture diariste apparaît ici comme un acte de dissidence et le retrait dans le journal comme le choix d'un exil symbolique et critique. Nous avons arrêté le premier tome en 1857 parce que c'est le moment où l'on entre dans les échanges littéraires, où des relations se créent avec les milieux littéraires. En 1858, la mutation s'est accomplie : les Goncourt se voient désormais comme appartenant au monde des Lettres, ils ne sont plus à la marge, la rupture avec la bohème est sur le point de s'accomplir. Après 1870, et consécutivement aussi à la mort de Jules, le Journal change progressivement. Il ne va plus être ponctué par des aphorismes, et les proses poétiques ne sont plus de la même nature. Elles deviennent nostalgiques, évocation d'un temps passé. L'humour se fait plus rare, la rosserie cesse souvent d'être spirituelle. Cela ne signifie pas qu'Edmond a moins de brio 
que son frère, mais on peut penser que l'écriture duelle constituait une sorte d'entraînement à la causticité et qu'elle est productrice d'ironie, de brillance, de verve, de bons mots. Par ailleurs, à partir d'un certain moment, Edmond veut apparaître comme le patriarche des Lettres, et la construction préposthume de cette figure infléchit le style du Journal.

F. S.-T. - Par l'édition de 1887 (Charpentier), Edmond a voulu faire du journal un livre. Peut-on aisément reconstituer les raisons (censure, etc.) des choix qu' il a opérés dans le manuscrit et des éventuelles réécritures? Possède-t-on des documents de genèse de l'édition commencée en 1887 ? Comment Edmond a-t-il procédé à partir du journal original pour faire le montage qu'il publie : a-t-il eu recours à un copiste, copié lui-même? Y a-t-il dans le manuscrit des marques d'un travail préparatoire à l'édition, coups de crayon ou autres?

J.-L.C. - Le travail pour l'édition Charpentier n'a pas laissé dans le manuscrit de traces explicites. J'ai repéré des sigles qui me sont incompréhensibles, par exemple des croix dans les marges qui restent mystérieuses, mais elles ne renvoient pas à une anthologie. Il est évident qu'Edmond a normalisé son journal pour l'édition. Il a censuré certains passages de manière à ne pas blesser ses contemporains, il n'a pas repris ce qui était trop cru. Il a procédé à un toilettage stylistique, rabotant ce que le texte pouvait avoir d'impromptu. Sans doute a-t-il également privilégié un certain type d'entrées, celles qui se rapportaient aux réseaux littéraires. Il me semble également que le toilettage du manuscrit en vue de l'édition Charpentier a eu une influence sur l'écriture par Edmond du journal mais peut-être aussi de ses romans. Dans les dernières années de tenue du journal, on observe une manière de continuité entre ce qui est écrit et ce qui est publié : le journal manuscrit des dernières années devient plus « lisse » d'un point de vue stylistique.

F. S.-T. - Le journal manuscrit constitue donc l'avant-texte de l'édition Charpentier. Est-il également un texte matriciel pour l'ouvre romanesque?

J.-L. C. - Je serais tenté de dire qu'il s'agit de deux textes différents, et qu'il faut considérer pour eux-mêmes. Très tôt, en effet, le journal, dans sa forme manuscrite, est conçu pour être publié tel quel. Je songe à la sténographie des propos de Sainte-Beuve, le 22 novembre 1862, qui sont accompagnés d'un commentaire significatif : «Et moi, je pensais que j'allais écrire pour l'avenir, aussi, ce qu'il me disait là et ce qu'il croyait tomber dans le vide, dans le néant, dans l'oubli, dans une oreille, et non dans ce livre. » Le manuscrit du journal est effectivement un texte-réservoir, le point de départ de fictions, une machine à produire des scénarios. L'on note dans le journal des injonctions programmatiques qui renvoient aux ouvrages de fiction. Le journal est en partie matriciel pour Idées et sensations, texte publié en 1866 chez Lacroix, qui se présente comme un ensemble de descriptions de paysages, d'anecdotes et $\mathrm{d}$ 'aphorismes et dont la matière est en grande partie empruntée au journal ou au Carnet de voyage en Italie : néanmoins, les extraits repris sont décontextualisés puisque la date des entrées est supprimée. Il faudrait étudier ce savant montage, qui n'est jamais chronologique. Dans Charles Demailly (1868), le chapitre XVII est intégralement constitué par le journal du héros. Or il s'agit d'un montage effectué à partir d'emprunts au Journal écrit par les deux frères. En ce qui concerne des romans rédigés par Edmond, comme La Faustin (1882) ou Chérie (1884), on constate que l'aîné des deux frères vient puiser des réserves mimétiques dans le Journal écrit antérieurement à deux. Pour m'en tenir à la seule année 1858 , les entrées du 13 , du 25 et du 26 février, du 11 avril, du 21 mai, de juillet (sans indication précise de jour), du 13 décembre ont alimenté les chapitres II, V, VI, VII, XLI de La Faustin en petits faits, en anecdotes, en expressions imagées, voire en personnages secondaires. Le manuscrit du journal est une mémoire dont le roman ordonne un faisceau de souvenirs en les présentifiant dans la fiction comme des pilotis du vraisemblable.

F. S.-T. - Comment se présentent les manuscrits des romans des Goncourt?

J.-L. C. - Les manuscrits des romans écrits en commun par les deux frères ont été brûlés, à l'exception de celui de Manette Salomon et de Madame Gervaisais. Les manuscrits de Chérie et des Frères Zemganno sont dans une collection privée ; les carnets préparatoires de La Fille Élisa 
et de Madame Gervaisais ont été étudiés, l'un par Robert Ricatte, l'autre par Marc Fumaroli. Un plan de Chérie, tout à fait significatif sur la manière de travailler d'Edmond, pouvait être consulté naguère chez un collectionneur privé. On trouve à la Bibliothèque nationale un dossier qui rassemble certaines des lettres de femmes reçues par Edmond, à la suite de l'appel qu'il avait lancé dans $L a$ Faustin (1881) : Edmond demandait à ses lectrices de lui adresser leurs souvenirs sur leur vie de jeune fille. Un collectionneur comme René Gimpel possédait des notes préparatoires à L'Art du XVIII' siècle ainsi que des notations prélevées à partir de la lecture de la Gazette des tribunaux pour La Fille Élisa. Il possédait aussi le carnet étudié par
Robert Ricatte, et d'autres documents encore. En ce domaine, un travail considérable reste à faire. Nous nous proposons avec Pierre Dufief, dans le cadre du séminaire Goncourt, d'étudier d'un point de vue génétique les romans écrits en commun par les deux frères, ce qui implique un retour sur les carnets préparatoires dispersés. Mais on voit déjà que l'approche philologique du Journal débouche de manière quasi inéluctable sur des questions qui ressortissent à la génétique. Nous en sommes, en cette matière, à des balbutiements. Mais il faudra bien procéder à un dépassement de la philologie pour comprendre à quel point cette œuvre passionnante, comédie humaine du monde des lettres pour le demi-siècle, est un véritable texte. 